\title{
Article \\ Machine Learning Based Low-Cost Optical Performance Monitoring in Mode Division Multiplexed Optical Networks
}

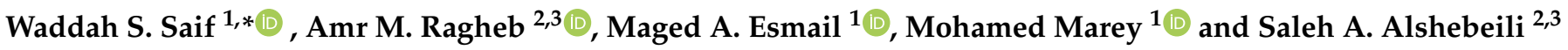 \\ 1 Smart Systems Engineering Laboratory, College of Engineering, Prince Sultan University, \\ Riyadh 11586, Saudi Arabia; mesmail@psu.edu.sa (M.A.E.); mfmmarey@psu.edu.sa (M.M.) \\ 2 Department of Electrical Engineering, King Saud University, Riyadh 11421, Saudi Arabia; \\ aragheb@ksu.edu.sa (A.M.R.); dsaleh@ksu.edu.sa (S.A.A.) \\ 3 KACST-TIC in Radio Frequency and Photonics for the e-Society, King Saud University, \\ Riyadh 11421, Saudi Arabia \\ * Correspondence: waddah.alkhulidy@gmail.com
}

check for updates

Citation: Saif, W.S.; Ragheb, A.M.; Esmail, M.A.; Marey, M.; Alshebeili, S.A. Machine Learning Based Low-Cost Optical Performance Monitoring in Mode Division Multiplexed Optical Networks. Photonics 2022, 9, 73. https:// doi.org/10.3390/photonics9020073

Received: 30 December 2021

Accepted: 26 January 2022

Published: 28 January 2022

Publisher's Note: MDPI stays neutral with regard to jurisdictional claims in published maps and institutional affiliations.

Copyright: (C) 2022 by the authors. Licensee MDPI, Basel, Switzerland. This article is an open access article distributed under the terms and conditions of the Creative Commons Attribution (CC BY) license (https:// creativecommons.org/licenses/by/ $4.0 /)$.

\begin{abstract}
Real-time optical performance monitoring (OPM) is of the utmost importance in adaptive optical networks to enable awareness of channel conditions and to achieve high quality of service. In single-mode fiber (SMF)-based networks, optical signal-to-noise ratio (OSNR) and chromatic dispersion (CD) monitoring have been extensively studied in the literature. In this work, we consider OPM in few-mode fiber (FMF) networks employing non-coherent detection. OPM in such networks is a challenging task, as FMF has an additional performance-limiting impairment over SMF, namely mode coupling (MC). Here, we propose an OPM scheme to estimate three FMF channel parameters: OSNR within the range of 8 to $20 \mathrm{~dB}, \mathrm{CD}$ within the range of 160 to $1120 \mathrm{ps} / \mathrm{nm}$, and different levels of MC. The proposed scheme uses a stacked auto-encoder (AE) to extract features with reduced dimensionality compared to the original data. These features are used to train an artificial neural network (ANN) regressor. Simulation results show that the proposed OPM scheme can accurately estimate the OSNR, CD, and MC with root mean square error (RMSE) values of $0.0015 \mathrm{~dB}, 0.28 \mathrm{ps} / \mathrm{nm}$, and $7.88 \times 10^{-6}$, respectively. The performance of proposed OPM scheme is also evaluated against different types of features commonly used in literature.
\end{abstract}

Keywords: few mode fiber; optical performance monitoring; machine learning

\section{Introduction}

Mode-division multiplexing (MDM) is a technology to alleviate the capacity crunch caused by the bandwidth-hungry applications evolved around the single-mode optical networks [1,2]. Few-mode fiber (FMF) is an example of such a technology to increase system's spectral efficiency, where each guided mode traveling through the fiber is considered a different optical channel [1]. On the other hand, it is envisaged that optical communication networks will evolve from fixed to adaptive architectures [3,4]. In adaptive networks such as an elastic optical network (EON), the optical network fiber link state will be heterogeneous and dynamic, and the network architecture will be more complex $[3,5]$. The transmitted optical signals are vulnerable to several transmission impairments, which reduce network reliability and stability. Therefore, it is essential to obtain continuous and real-time information about channel impairments to automatically adjust network parameters such as optical power, symbol rate, modulation format (MF), etc. Optical performance monitoring (OPM) is an indispensable tool in such networks as it provides flexibility and improves networks controllability [6,7]. The OPM module can estimate different network parameters such as optical signal-to-noise ratio (OSNR), chromatic dispersion (CD), and optical power from the physical layer. These parameters help managing dynamic fiber optic communication networks [8]. 
Machine learning (ML) algorithms have received significant attention in the communications field [9]. For instance, International Telecommunication Union (ITU) has defined unified architecture for ML functions and their interface in 5G and future networks [10]. Along the same lines, the adoption of ML in optical communications, particularly OPM, is driven by a large number of adjustable parameters that are enabled by the increased complexity and dynamism of next-generation optical communication [11-13]. ML algorithms have shown superior results when handling non-linear problems. In addition, recent advances in ML techniques (i.e., deep learning (DL)) can solve complicated models without the need to develop extensive analytical models, which makes them suitable to meet the challenge of monitoring multiple impairments in adaptive and heterogeneous optical networks [12].

Several ML-based OPM approaches have been presented to estimate optical parameters such as OSNR, CD, polarization mode dispersion (PMD), phase noise (PN), etc. For instance, the authors in [14] exploited a convolutional neural network (CNN) to extract features from raw data. This technique does not require manual feature extraction. However, this technique is focused on estimating OSNR values. Another OPM scheme-based long-short term memory (LSTM) is introduced in [15]. The LSTM is trained using fast Fourier transfer (FFT) features to simultaneously estimate OSNR and nonlinear noise power. This technique is robust to fiber nonlinearity. However, FFT-based features do not take full advantage of LSTM, which is well-suited for time-dependency data. In [16], the authors demonstrated joint OSNR monitoring and MF identification using an artificial neural network (ANN). The cumulative distribution function (CDF) after mapping the received signal onto Stokes space planes is used as input to the ANN. The authors in [17] demonstrated OSNR monitoring and MF identification scheme using binary-CNN (B-CNN) with constellation diagram in which the activation weights were constrained to $+/-1$. The computational complexity of this method is relatively low compared to the traditional CNN. However, it is more representative to utilize a CNN regression rather than a classifier when estimating continuous OSNR values. Besides, the OPM scheme based on asynchronous in-phase quadrature histogram $(\mathrm{IQH})$ features with a support vector machine has been demonstrated in [18] to monitor OSNR, CD, PMD, and PN. This technique is insensitive to PN (preserve the phase information). However, the IQH features are constructed from the statistics of both I and Q samples.

The above OPM techniques require coherent acquisition systems. However, these systems are too expensive and inappropriate for intermediate nodes spread along optical networks. Therefore, cost-effective OPM systems (i.e., direct detection) are more feasible. The majority of ML-based OPM techniques for direct detection methods rely on extracting statistical features from the received signal. These features are employed for training ML algorithms. Asynchronous amplitude histograms (AAH) [19-21] and asynchronous tap delay histogram (ADTH) [22-27] are the most common features of OPM. AAH is obtained by sampling the received signal at a speed lower than the sampling rate. AAH is costeffective but only provides information about the signal's amplitude, which degrades its performance when dealing with impairments that require information on the slope, such as CD. On the other hand, ADTH provides some information about the slope of the signal (i.e., phase portraits) by sampling at two different time instants with a certain delay between them. There are many ML algorithms trained using these features to perform OPM, such as ANN [19,22,23], principal component analysis (PCA) [24], DNN [27], DNN with multi-task learning [20,25,26,28], and DNN with transfer learning [21].

So far, the use of OPM for single mode fiber (SMF)-based networks has received much attention in the literature. However, adapting OPM for FMF networks is still in its early stages. Compared to SMF, the optical signal in FMF has other impairments, such as cross-talk between different modes (Mode coupling (MC)), which makes the monitoring process more challenging. In [29], we studied OPM in FMF-based coherent optical networks. However, the use of coherent acquisition increases the cost of monitoring systems. In this work, direct-detection technique is considered, which is cost-effective compared to coherent 
detection. In our development, three different impairments are monitored, which include OSNR, CD, and MC in FMF-based optical networks. The proposed monitoring technique relies on the use of stack auto-encoder (AE) to extract representative features with reduced dimensionality compared to the input features data (i.e., ADTH in our case). The reduceddimensionality features are then used as inputs to the ANN regressor to perform OPM. Extensive simulations have been conducted to consider five spatial modes (i.e., $\mathrm{LP}_{01}, \mathrm{LP}_{11 \mathrm{a}}$, $\mathrm{LP}_{11 \mathrm{~b}}, \mathrm{LP}_{21 \mathrm{a}}$, and $\mathrm{LP}_{21 \mathrm{~b}}$ ) with dual polarization-quadrature phase shift keying (DP-QPSK) and 10 Gbaud transmission speed. We assess the effectiveness of the proposed technique by evaluating the OPM performance under different channel conditions. Furthermore, we show the performance of an ANN regressor when the proposed features are being used in comparison with the utilization of AAH and ADTH features commonly used in the literature.

The structure of this paper is as follows: Section 2 provides brief description about FMF optical channel. Section 3 introduces the proposed OPM technique. Section 4 gives details of simulation setup. Section 5 presents the simulation results with discussion. Finally, Section 6 provides concluding remarks.

\section{Fmf Optical Channel}

FMF supports a few guided modes, each of which is treated as an individual channel. These modes are characterized as linearly polarized $\mathrm{LP}_{l p}$ waves, where $l$ and $p$ are integer numbers specifying the radial and azimuthal indices. The guided modes intensity for a given $\mathrm{LP}_{l p}$ can be represented mathematically as $[30,31]$

$$
\psi(r, \phi)=\left\{\begin{array}{l}
F_{l p}(r) \cos (n \phi) \\
F_{l p}(r) \sin (n \phi)
\end{array}\right.
$$

where $r$ denotes the distance from the center core (e.g., at the center $r=0$ ) and $F$ is the mode field. SMF has a small core, only the fundamental mode $\mathrm{LP}_{01}$ propagates across the fiber. Increasing the fiber core allows higher-order modes such as $\mathrm{LP}_{11}, \mathrm{LP}_{21}$, etc., to pass through the fiber. These modes propagate in two different spatial orientations. The two spatial configurations are represented by $\cos ($.$) and \sin ($.$) .$

Ideally, the guided modes in the FMF channel propagate in an orthogonal way. However, in reality, there are some energy exchanges (i.e., MC) between the different modes. The MC can be induced duo to fiber stress/bend, misaligned connectors, etc. The MC between the different spatially multiplexed modes are illustrated in Figure 1, where the diagonal elements denote the self-coupling coefficients while the remaining elements denote the cross-coupling coefficients.

\begin{tabular}{|c|c|c|c|c|c|}
\hline & $\mathrm{LP}_{01}$ & $\mathrm{LP}_{11 \mathrm{a}}$ & $\mathrm{LP}_{11 \mathrm{~b}}$ & $\mathrm{LP}_{21 \mathrm{a}}$ & $\mathrm{LP}_{21 \mathrm{a}}$ \\
\hline $\mathrm{LP}_{01}$ & 1 & 0 & 0 & 0 & 0 \\
\hline $\mathrm{LP}_{11 \mathrm{a}}$ & 0 & 1 & 0 & 0 & 0 \\
\hline lb & 0 & 0 & 1 & 0 & 0 \\
\hline $\mathrm{LP}_{21 \mathrm{a}}$ & 0 & 0 & 0 & 1 & 0 \\
\hline $\mathrm{LP}_{21 \mathrm{a}}$ & 0 & 0 & 0 & 0 & 1 \\
\hline
\end{tabular}

(a)

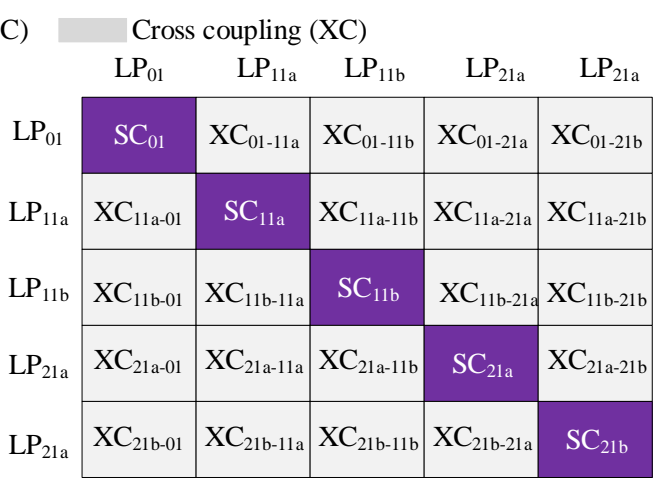

(b)

Figure 1. Coupling matrix for the five spatial modes: (a) Ideal FMF transmission, (b) FMF transmission in the presence of MC. 


\section{The Proposed OPM Scheme}

FMF-based optical networks transmit data over different orthogonal fiber modes, each mode being an independent transmission channel. In such a setting, MC arises which may destroy the orthogonality between modes. Therefore, it is very important to monitor MC as well as OSNR and CD in FMF-based elastic optical networks. If left unchecked, they can cause significant degradation of performance. The network should know the channel conditions in real time so that it can make appropriate routing decisions, diagnosing the network and repairing the damage, driving a compensator/equalizer, amplifying the signal, changing modulation format, etc. Therefore, it is important to build an accurate OPM model which helps to optimize the network parameters. In the following subsections, we discuss in detail the proposed OPM-based ADTH with AE.

\subsection{Asynchronous Tap Delay Histogram (ADTH)}

In the next-generation optical networks, OPMs modules should be built along the transmission optical link. These modules should be cost-effective utilizing low-cost devices with an asynchronous and low speed sampler. AAH or ADTH can be utilized for transmission link monitoring at the intermediate nodes. AAH provides only information about the signal amplitude. However, ADTH delivers rich information from one more sampling component (i.e., extracts information about the slope of the received signal). In ADTH, the received signal is sampled asynchronously in pairs $\left(u_{i}, v_{i}\right)$ where a constant delay $\Delta t$ between these sampling pairs is used, as shown in Figure 2a. The acquired $\left(u_{i}, v_{i}\right)$ is used to build a 2 dimensional (2D) histogram, which is called ADTH (see Figure 2b). It is worth noting that ADTH is constructed from signal's samples with no a prior knowledge of symbol rate [32].
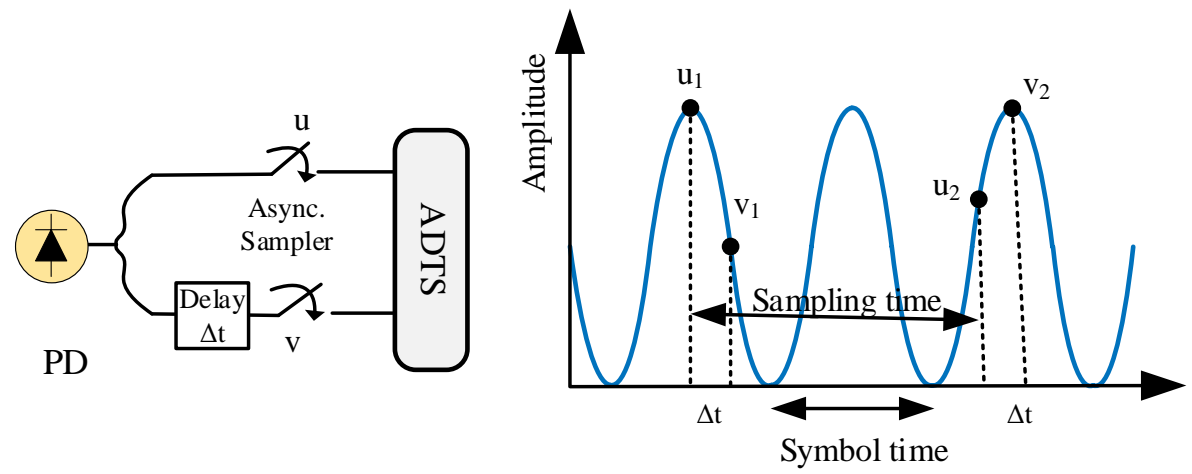

(a)

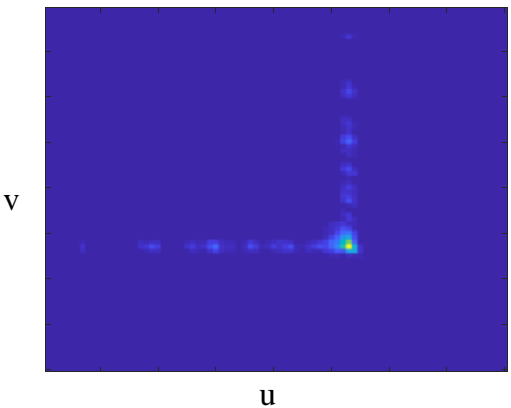

(b)

Figure 2. The principle of ADTH: (a) the amplitude of sampled signal, and (b) the generated ADTH.

In this work, we used ADTH to monitor fiber impairments in FMF-based networks including OSNR, CD, and MC. Figure 3 shows ADTH plots of $\mathrm{LP}_{11 \mathrm{a}}$ mode of DP-QPSK 
signal with different values of MC. It is clear from Figure 3 that there are clear differences between the ADTHs plots, which enable effective monitoring of MC.

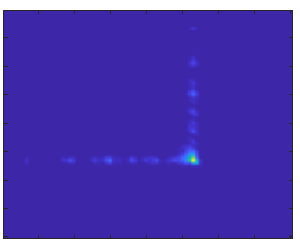

(a)

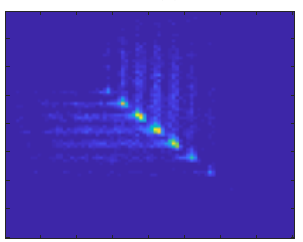

(e)

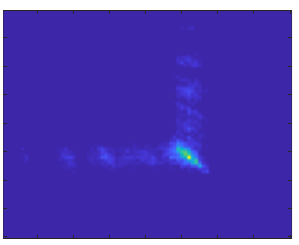

(b)

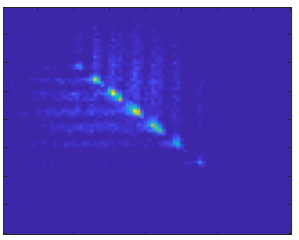

(f)

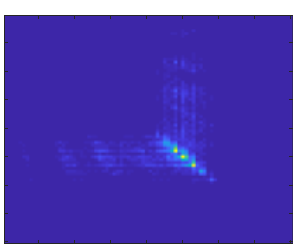

(c)

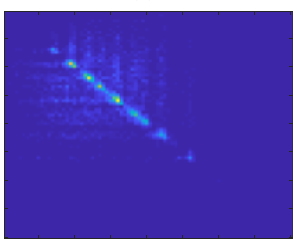

(g)

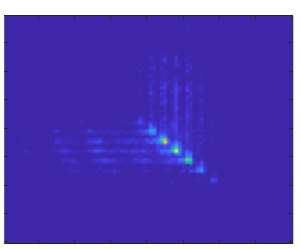

(d)

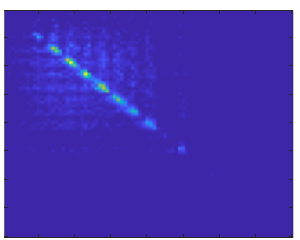

(h)

Figure 3. ADTH features at different levels of $\mathrm{MC}$ impairment: (a) $\mathrm{MC}=0.96$, (b) $\mathrm{MC}=0.88$, (c) $\mathrm{MC}=0.81,(\mathbf{d}) \mathrm{MC}=0.74,(\mathbf{e}) \mathrm{MC}=0.67,(\mathbf{f}) \mathrm{MC}=0.62,(\mathrm{~g}) \mathrm{MC}=0.53$, and $(\mathbf{h}) \mathrm{MC}=0.47$.

\subsection{Opm Using ADTH with AE}

The ADTH features are represented in a 2D form of size $(k \times k)$, where $k$ is a positive integer. The ADTH features can be concatenated in the form of a 1D vector $\mathbf{x}$ with length $N=k^{2}$. AE is exploited here to extract features from the 1D vector $\mathbf{x}$. These features are then used as inputs to an ANN regressor, as shown in Figure 4. The AE is a form of unsupervised feedforward neural network, where the number of input layers is equal to the number of output layers, and designed so that its output matches its input [33]. The main goal of AE is to produce a reduced set of features vector $\mathbf{z}$ that sufficiently represents the input vector $\mathbf{x}$. This can be achieved by setting the number of neurons in the hidden layer less than that in the input layer. Basically, the AE consists of two parts:

- An encoder part which transfers the input vector $\mathbf{x}$ to a feature vector $\mathbf{z}$.

- A decoder part that uses the feature vector $\mathbf{z}$ to reconstruct the input vector $\mathbf{x}$.

Consider an input vector $\mathbf{x} \subset \mathbf{R}^{N}$. The encoder maps the input $\mathbf{x}$ to the vector $\mathbf{z} \subset \mathbf{R}^{n}$, where $N \gg n$, as follows.

$$
\mathbf{z}=f^{(1)}\left(\mathbf{W}^{(1)} \mathbf{x}+\mathbf{b}^{(1)}\right)
$$

where $\mathbf{W}^{(1)} \subset \mathbf{R}^{N \times n}$ represents the weight matrix, $\mathbf{b}^{(1)}$ is the bias vector, superscript (1) denotes the hidden layer, and $f$ is the activation function. The decoder maps the vector $\mathbf{z}$ into the vector $\mathbf{x}^{\prime}$ which represents the approximation of input vector $\mathbf{x}$, as follows.

$$
\mathbf{x}^{\prime}=f^{(2)}\left(\mathbf{W}^{(2)} \mathbf{z}+\mathbf{b}^{(2)}\right)
$$

The most common activation function utilized for both encoder $f^{(1)}(\sigma)$ and decoder $f^{(2)}(\sigma)$ is the logistic sigmoid function which is represented as [33]

$$
f(\sigma)=\frac{1}{1+e^{-\sigma}}
$$

The cost function for the training process is given as [34]

$$
L=\frac{1}{M} \sum_{i=1}^{M} \sum_{j=1}^{N}\left(x_{i j}+x_{i j}^{\prime}\right)^{2}+\lambda \Omega_{w}+\beta \Omega_{S}
$$

where $M$ is the number of training realizations, $N$ is the number of samples, $\Omega_{w}$ and $\Omega_{S}$ represent the weight decay constraint and sparsity constraint, respectively. The idea behind these constraints is to maintain the average activation value of each neuron in the hidden 
layer close to zero. The coefficients $\lambda$ and $\beta$ are specified by the user to control the impact of these constraints. The weight decay $\Omega_{w}$ and sparsity $\Omega_{S}$ constraints are given as [34]

$$
\begin{gathered}
\Omega_{w}=\frac{1}{2} \sum_{i=1}^{M} \sum_{j=1}^{N}\left(w_{i j}\right)^{2} \\
\Omega_{s}=\sum_{i=1}^{n} K L(\rho \| \hat{\rho})=\sum_{i=1}^{n} \rho \log \left(\frac{\rho}{\hat{\rho}}\right)+(1-\rho) \log \left(\frac{1-\rho}{1-\hat{\rho}}\right)
\end{gathered}
$$

where $K L$ is the Kullback-Leibler divergence which measures how different the average activation value, $\hat{\rho}$, of a neuron $i$ from its desired value, $\rho$. The average activation, $\hat{\rho}$, is given as

$$
\hat{\rho}_{i}=\frac{1}{M} \sum_{j=1}^{M} z_{i} x_{j}=\frac{1}{M} \sum_{j=1}^{M} f\left(w_{i}^{T} x_{j}+b_{i}\right)
$$

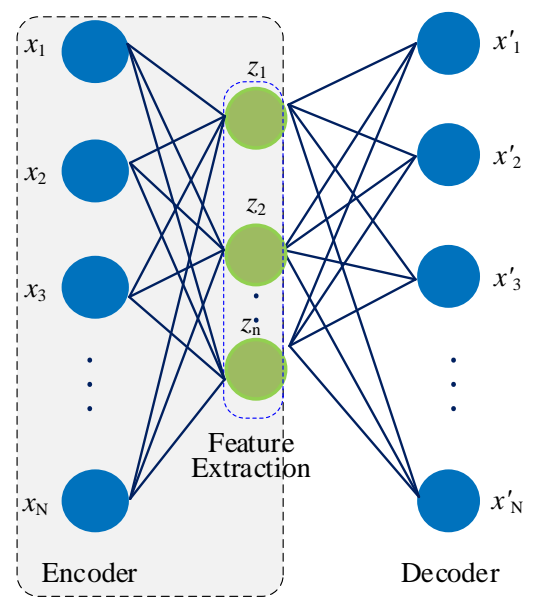

(a)

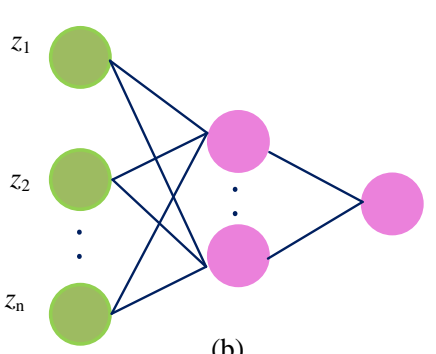

(b)

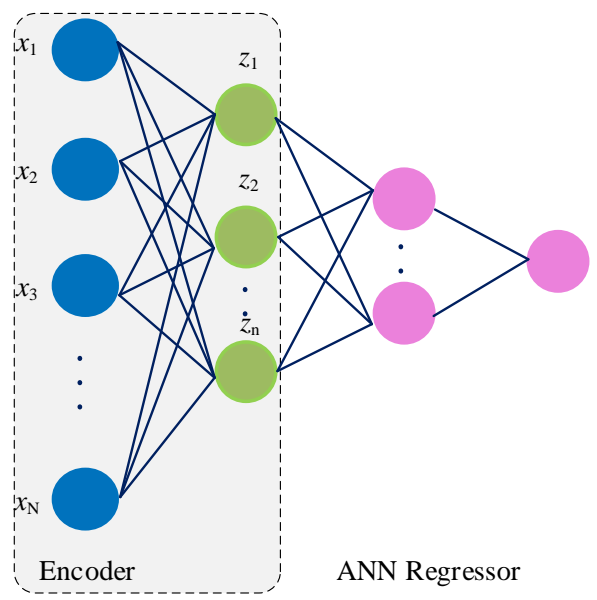

(c)

Figure 4. (a) Schematic diagram of AE, (b) ANN regressor, and (c) stacked encoder with an ANN regressor.

After the AE training is completed, the extracted features (vector $\mathbf{z}$ ) are inputted to an ANN regressor which has a single hidden layer with sigmoid activation function followed by an output layer of one neuron, as shown in Figure $4 \mathrm{~b}$. The ANN regressor is trained to estimate the value of different impairments in supervised manner. Backpropagation is utilized to update the weight and bias values based on the mean square error (MSE) between the output $d_{o}$ and target $d_{t}$. That is,

$$
M S E=\frac{1}{M} \sum_{j=1}^{M}\left|d_{0}^{(j)}-d_{t}^{(j)}\right|^{2}
$$

Finally, the encoder part is stacked with the ANN regressor and re-trained together in order to fine tune the weights and biases for the whole system. Once the training is 
completed, the system will be ready to use in the testing phase in the form shown in Figure $4 \mathrm{c}$.

\section{Simulation Setup}

We used VPI TransmissionMaker ${ }^{\mathrm{TM}}$ software, version 11, for modeling the few-mode fiber system. VPI is a commercial tool widely used to model different types of photonic components, devices, and systems [35]. The simulation setup of the proposed OPM based FMF with five spatial modes is shown in Figure 5. At the transmitter side, we generated five DP-QPSK signals with transmission speed of 10 Gbaud. These signals are converted into five spatial modes including $\mathrm{LP}_{01}, \mathrm{LP}_{11 \mathrm{a}}, \mathrm{LP}_{11 \mathrm{~b}}, \mathrm{LP}_{21 \mathrm{a}}$, and $\mathrm{LP}_{21 \mathrm{~b}}$ via spatial multiplexer, and then fed to the FMF channel. The dispersion coefficients of the different modes are, $21.1 \mathrm{ps} /(\mathrm{nm} \cdot \mathrm{km}), 22.0 \mathrm{ps} /(\mathrm{nm} \cdot \mathrm{km})$, and $21.4 \mathrm{ps} /(\mathrm{nm} \cdot \mathrm{km})$, for $\mathrm{LP}_{01}, \mathrm{LP}_{11}, \mathrm{LP}_{21}$, respectively. The fiber length is adjusted to provide CD in the range of 160 to $1120 \mathrm{ps} / \mathrm{nm}(160 \mathrm{ps} / \mathrm{nm}$ step size). To emulate the effect of $\mathrm{MC}$, we consider two non-ideal couplers with lateral misalignment D $=0.05,0.15$ and 0.25 , as demonstrated in Figure 5.

At the receiver side, an FMF de-multiplexer is employed to convert the five spatial modes into five separate signals. An erbium-doped fiber amplifier (EDFA) is utilized to add amplified spontaneous emission (ASE) into the signal. To adjust the value of OSNR, an optical amplifier with a variable optical attenuator is used. The OSNR is varied within the range of 8-20 dB. Finally, each signal is fed into photodiode which converts the optical signal into an electrical signal. Then, the signal is asynchronously sampled using pairs of samplers to obtain 8192 amplitude samples. These samples are then stored for offline processing, which includes data preprocessing (normalization), features extraction, and implementation of machine learning algorithms. Offline processing is performed using Matlab software. In particular, we applied sample normalization by division over sample's standard deviation. Then, we built the ADTH and AAH features, which are used as inputs to the ML algorithms.

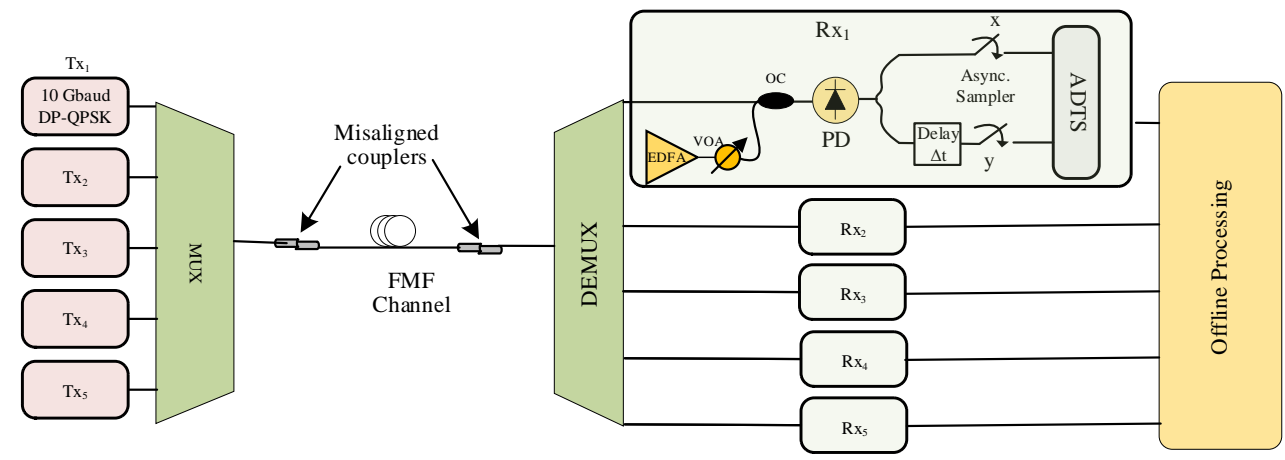

Figure 5. Simulation setup.: MUX: multiplexer, EDFA: erbium-doped fiber amplifier, VOA: variable optical attenuator, DEMUX: de-multiplexer, FMF: few mode fiber, PD: photodetector. ADTH: asynchronous tap delay histogram.

\section{Results and Discussion}

In this work, we consider the MC caused by lateral misalignment of two connectors [36]. To avoid redundancy, we only show the OPM results of $\mathrm{LP}_{11 \mathrm{a}}$. For the purpose of ML training and testing, we constructed a dataset that includes three impairments: OSNR values within the range of 8 to $20 \mathrm{~dB}$ in $2 \mathrm{~dB}$ step size, CD within the range from 160 to $1120 \mathrm{ps} / \mathrm{nm}$ with step of $160 \mathrm{ps} / \mathrm{nm}$, and a list of self-coupling values of $0.96,0.88,0.81,0.74$, $0.67,0.62,0.53$, and 0.47 . For each impairment, we generated 100 independent realizations. We split the dataset into $70 \%$ for training and the rest for testing. The monitoring result is averaged over 25 independent runs, where for each run, different training and testing data sets are selected randomly.

The monitoring results are obtained using three features, each of which was used as an input to the ANN regressor. The first and second features are AAH and ADTH which have 
been extensively used in literature, see [12] and the references therein. The third feature is the proposed ADTH-AE obtained by applying the AE to the ADTH input vector $\mathbf{x}$.

At the beginning, we conducted simulation results using the training data to obtain the optimal numbers of histograms bins of AAH and ADTH features, and the optimal number of neurons for both AE and ANN regressors. The optimal numbers of these parameters are determined by varying the number of histogram bins and number of neurons and observing the monitoring accuracy for the different impairments (i.e., OSNR, CD, MC). We consider the RMSE metric as a quantitative measure. It is worth mentioning that only one hidden layer is considered for both $\mathrm{AE}$ and $\mathrm{ANN}$ regressor. We noticed that using more than one hidden layer does not improve the results. For the AAH features with ANN regressor, the monitoring accuracy is evaluated at different numbers of AAH bins $(5,10,20$, $50,80,100,150$, and 200) and at different numbers of ANN hidden nodes $(5,10,20,50)$.

Figure 6 a shows the monitoring results of OSNR, CD, and MC in terms of RMSE in the $y$-axis and number of neurons of ANN regressor in $\mathrm{x}$-axis at different numbers of AAH bins. It can be seen from Figure 6 a that the minimum RMSE values for OSNR, CD, and MC are achieved with number of bins (neurons) as follows: 50(10), 50(10), and 50(20). Accordingly, we considered the AAH with 50 bins and ANN regressor with 10 neurons for the rest of our work. Similarly, we considered $(80,80)$ bins for the ADTH features and 10 neurons for the ANN regressor according to the results reported in Figure $6 \mathrm{~b}$. On the other hand, for ADTH-AE, we fixed the number of ADTH bins to the optimum value, $80 \times 80(k=80)$, and varied the number of neurons for AE and ANN regressor. It can be observed from Figure $6 \mathrm{c}$ that 100 neurons for AE and 10 neurons for ANN regressor are sufficient to achieve better accuracy for all types of impairments.
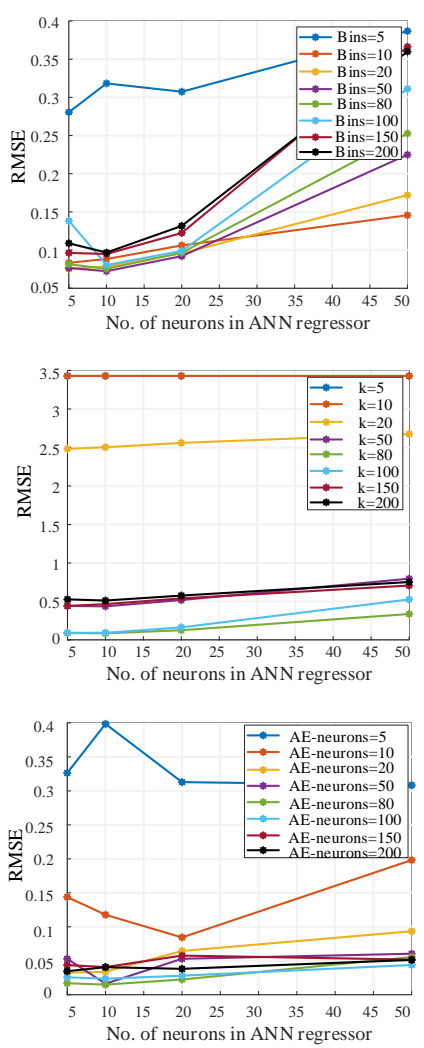

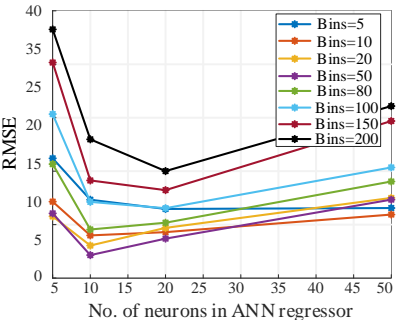

(a)

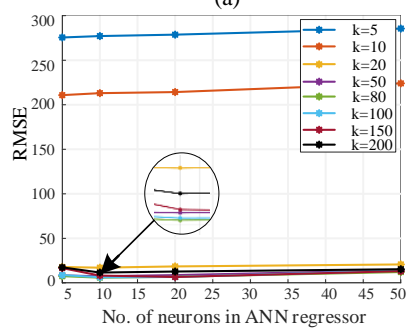

(b)

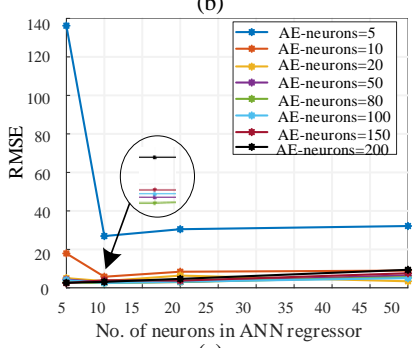

(c)
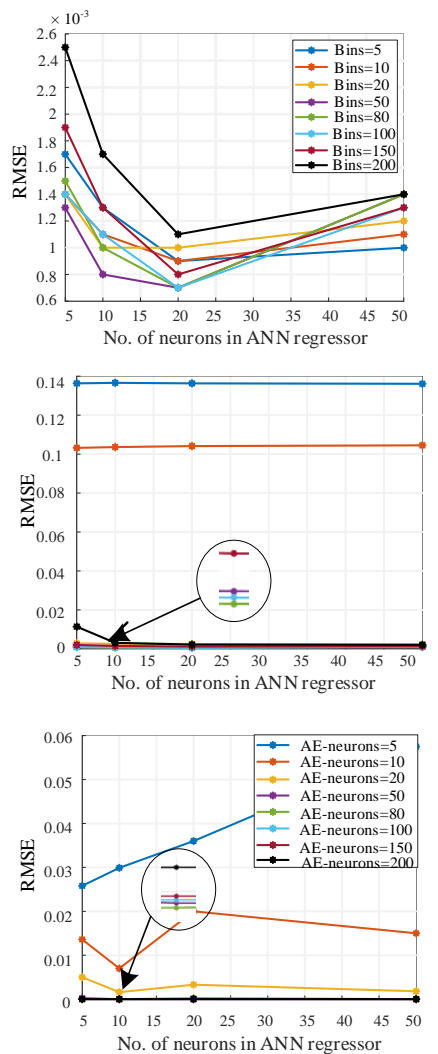

Figure 6. OPM optimization results of AAH (left), 1-D ADTH (middle), and AE-ADTH (right) with ANN regressor: (a) OSNR monitoring (b) CD monitoring, and (c) MC monitoring.

The test results of the OPM monitoring with the optimum parameters, as determined from the training phase, for of the three parameters including OSNR, CD, and MC are shown in Figure 7. For the OSNR monitoring, it is clear from Figure 7a that the ADTH- 
AE can successfully estimate the OSNR with average RMSE $0.0015 \mathrm{~dB}$. However, the performance for ADTH are slightly better than AAH, where the RMSE values for AAH and $\mathrm{ADTH}$ are 0.07 and $0.06 \mathrm{~dB}$, respectively. Similarly, for CD and MC monitoring, the ADTH-AE provides the better result when compared with that of the AAH and ADTH features (see Figure $7 \mathrm{~b}, \mathrm{c}$ ).

We also assess the proposed OPM algorithm on the presence of multiple impairments. We consider three cases: light, moderate, and severe, as illustrated in Table 1. For instance, we estimate the OSNR in the presence of three values of CD and MC. In the first case (light case), we consider low CD and MC values corresponding to $160 \mathrm{ps} / \mathrm{nm}$ and 0.98 , respectively. However, the moderate case corresponds to $\mathrm{CD}=550 \mathrm{ps} / \mathrm{nm}$ and $\mathrm{MC}=0.74$. Likewise, the severe case corresponding to $C D=1100 \mathrm{ps} / \mathrm{nm}$ and $\mathrm{MC}=0.47$. Other values could be chosen for the three cases but the idea here is to consider representative cases to examine the robustness of the proposed algorithm and to which extent reasonable results can be obtained.
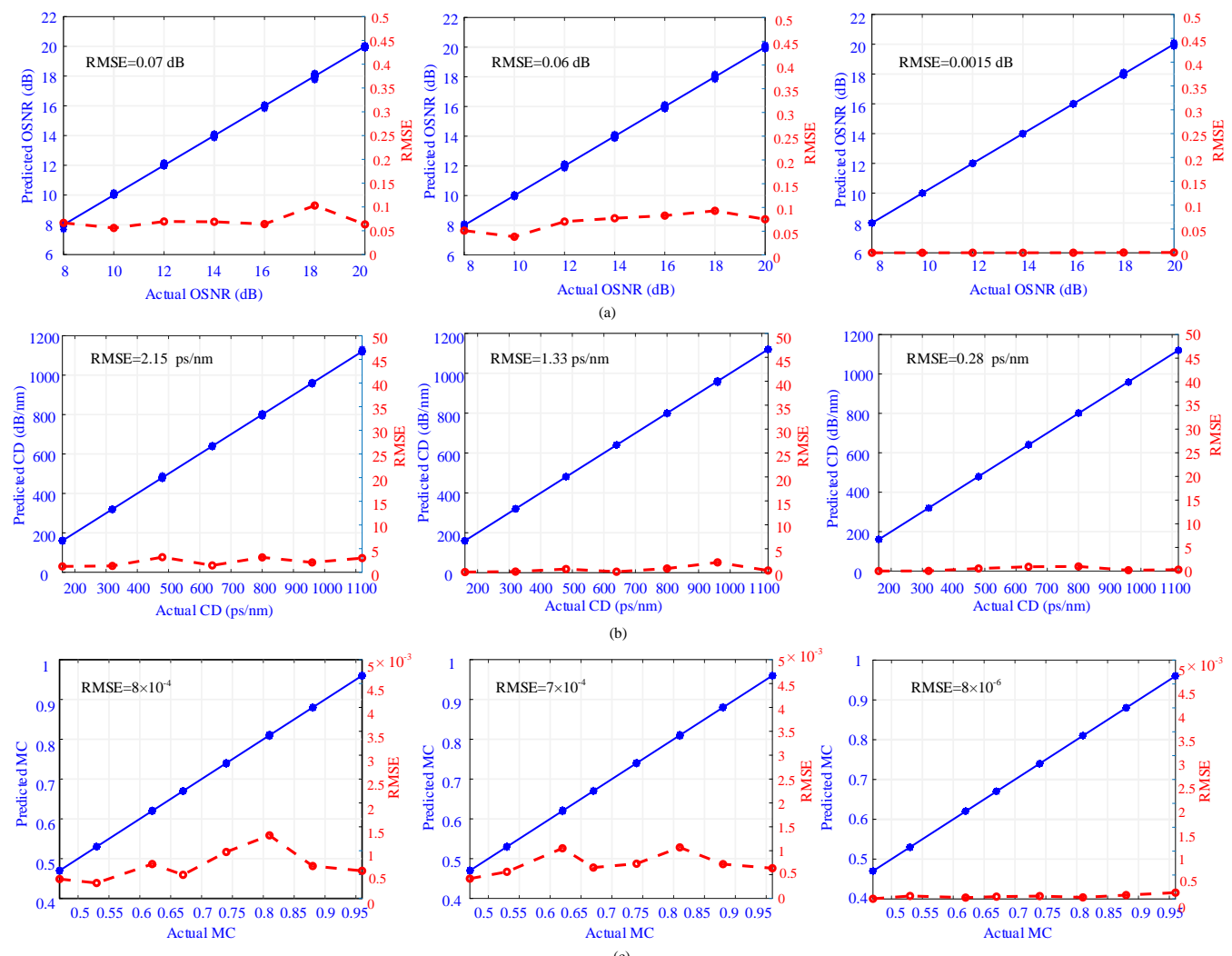

Figure 7. OPM results using AAH (left), 1-D ADTH (middle), and AE-ADTH (right) with ANN regressor: (a) OSNR monitoring (b) CD monitoring, and (c) MC monitoring.

Table 1. Scenarios for evaluating the performance of proposed OPM scheme.

\begin{tabular}{llll}
\hline & Case 1 (Light) & Case 2 (Moderate) & Case 3 (Severe) \\
\hline \multirow{2}{*}{ OSNR } & $\mathrm{CD}=160 \mathrm{ps} / \mathrm{nm}$ & $\mathrm{CD}=550 \mathrm{ps} / \mathrm{nm}$ & $\mathrm{CD}=1100 \mathrm{ps} / \mathrm{nm}$ \\
& $\mathrm{MC}=0.96$ & $\mathrm{MC}=0.74$ & $\mathrm{MC}=0.47$ \\
\hline $\mathrm{CD}$ & $\mathrm{OSNR}=20 \mathrm{~dB}$ & $\mathrm{OSNR}=14 \mathrm{~dB}$ & OSNR $=8 \mathrm{~dB}$ \\
& $\mathrm{MC}=0.96$ & $\mathrm{MC}=0.74$ & $\mathrm{MC}=0.47$ \\
\hline \multirow{2}{*}{$\mathrm{MC}$} & $\mathrm{OSNR}=20 \mathrm{~dB}$ & $\mathrm{OSNR}=14 \mathrm{~dB}$ & $\mathrm{OSNR}=8 \mathrm{~dB}$ \\
& $\mathrm{CD}=160 \mathrm{ps} / \mathrm{nm}$ & $\mathrm{CD}=550 \mathrm{ps} / \mathrm{nm}$ & $\mathrm{CD}=1100 \mathrm{ps} / \mathrm{nm}$ \\
\hline
\end{tabular}


The monitoring results of OSNR, CD, and MC using AAH, ADTH, and ADTH-AE for the three cases are summarized in Table 2. For OSNR monitoring, the ADTH-AE provides high accuracy results even in the presence of high values of $C D$ and MC (i.e., $\mathrm{CD}=1100 \mathrm{ps} / \mathrm{nm}$ and $\mathrm{MC}=0.47$ ), where the RMSE is of value $0.06 \mathrm{~dB}$. However, for $\mathrm{AAH}$ and ADTH, the RMSE increases to 0.17 and 0.16, respectively. Similarly, for MC monitoring, when OSNR $=8 \mathrm{~dB}$ and $\mathrm{CD}=1100 \mathrm{ps} / \mathrm{nm}$, the RMSE of ADTH-AE is $1 \times 10^{-4}$. Nevertheless, the AAH and ADTH almost have the same accuracy. On the other hand, for CD monitoring, the RMSE rises to $633 \mathrm{ps} / \mathrm{nm}$ and $389 \mathrm{ps} / \mathrm{nm}$ for AAH and ADTH, respectively. It can be observed that AAH and ADTH have approximately the same OSNR and $\mathrm{MC}$ monitoring results. Nonetheless, for $\mathrm{CD}$ monitoring, the ADTH outperforms the $\mathrm{AAH}$. This can be interpreted as CD causes signal broadening, and thus ADTH can capture some information about the signal slope. However, for OSNR and MC, their effect is only on the amplitude, which can be easily captured using the AAH feature.

Table 2. Comparisons between monitoring results in terms of RMSE of ADTH, AAH, and AE-ADTH.

\begin{tabular}{cccccccccc}
\hline & \multicolumn{4}{c}{ RMSE of OSNR (B) } & \multicolumn{2}{c}{ RMSE of CD (ps/nm) } & \multicolumn{3}{c}{ RMSE of MC } \\
\cline { 2 - 10 } & AAH & ADTH & ADTH-AE & AAH & ADTH & ADTH-AE & AAH & ADTH & ADTH-AE \\
\hline Case 1 (light) & 0.07 & 0.06 & 0.0015 & 2.15 & 1.33 & 0.28 & $8 \times 10^{-4}$ & $7 \times 10^{-4}$ & $7.88 \times 10^{-6}$ \\
\hline Case 2 (moderate) & 0.16 & 0.12 & 0.004 & 203 & 76 & 37 & $24 \times 10^{-3}$ & $23 \times 10^{-3}$ & $3.18 \times 10^{-5}$ \\
\hline Case 3 (severe) & 0.17 & 0.16 & 0.06 & 633 & 389 & 302 & $28 \times 10^{-3}$ & $26 \times 10^{-3}$ & $1 \times 10^{-4}$ \\
\hline
\end{tabular}

\section{Conclusions}

This paper studies, for the first time in the literature, the OPM in non-coherent FMFbased optical networks. An ML-based OPM approach has been considered for FMF-based optical networks. The performance of the proposed OPM approach is assessed in the presence of three impairments: OSNR within the range of 8 to $20 \mathrm{~dB}, \mathrm{CD}$ within the range of 160 to $1120 \mathrm{ps} / \mathrm{nm}$, and different levels of MC. The proposed monitoring technique used an autoencoder to extract features from ADTH. The new features take the form of a reduced size of a $1 \mathrm{D}$ vector, to facilitate the use of less complex machine learning algorithms. The extracted features were used to train an ANN regressor. The performance of the proposed ADTH-AE features is compared with the AAH and ADTH features, which are widely used in the development of OPM schemes for SMF-based optical networks. The results showed that for OSNR and MC monitoring, there is a slight superiority of ADTH compared to AAH features. Meanwhile, for CD monitoring, the results of ADTH features have pronounced improvement over the AAH results. On the other hand, the proposed ADTH-AE has shown performance outperforming all other features, even in the presence of high values of channel impairments. Therefore, the proposed technique can effectively be utilized for cost-effective deployment of OPM modules in FMF-based optical networks.

Author Contributions: Conceptualization, W.S.S. and S.A.A.; methodology, W.S.S. and S.A.A.; software, W.S.S. and S.A.A.; validation, W.S.S., A.M.R., M.A.E., M.M. and S.A.A.; investigation, W.S.S., A.M.R., M.A.E., M.M. and S.A.A.; writing-original draft preparation, W.S.S.; writing-review and editing, W.S.S., A.M.R., M.A.E., M.M. and S.A.A.; supervision M.M. and S.A.A.; funding acquisition, M.M. and S.A.A. All authors have read and agreed to the published version of the manuscript.

Funding: The authors extend their appreciation to the Deputyship for Research \& Innovation, Ministry of Education in Saudi Arabia for funding this research work through the project number (DRI-KSU-1088).

Institutional Review Board Statement: Not applicable.

Informed Consent Statement: Not applicable.

Data Availability Statement: Not applicable. 
Acknowledgments: The authors extend their appreciation to the Deputyship for Research \& Innovation, Ministry of Education in Saudi Arabia for funding this research work.

Conflicts of Interest: The authors declare no conflict of interest.

\section{References}

1. Saridis, G.M.; Alexandropoulos, D.; Zervas, G.; Simeonidou, D. Survey and evaluation of space division multiplexing: From technologies to optical networks. IEEE Commun. Surv. Tutor. 2015, 17, 2136-2156. [CrossRef]

2. Essiambre, R.J.; Kramer, G.; Winzer, P.J.; Foschini, G.J.; Goebel, B. Capacity limits of optical fiber networks. J. Lightwave Technol. 2010, 28, 662-701. [CrossRef]

3. Gerstel, O.; Jinno, M.; Lord, A.; Yoo, S.B. Elastic optical networking: A new dawn for the optical layer? IEEE Commun. Mag. 2012, 50, s12-s20. [CrossRef]

4. Jinno, M.; Ohara, T.; Sone, Y.; Hirano, A.; Ishida, O.; Tomizawa, M. Elastic and adaptive optical networks: Possible adoption scenarios and future standardization aspects. IEEE Commun. Mag. 2011, 49, 164-172. [CrossRef]

5. Chatterjee, B.C.; Sarma, N.; Oki, E. Routing and spectrum allocation in elastic optical networks: A tutorial. IEEE Commun. Surv. Tutor. 2015, 17, 1776-1800. [CrossRef]

6. $\quad$ Dong, Z.; Khan, F.N.; Sui, Q.; Zhong, K.; Lu, C.; Lau, A.P.T. Optical performance monitoring: A review of current and future technologies. J. Lightwave Technol. 2016, 34, 525-543. [CrossRef]

7. Wang, D.; Jiang, H.; Liang, G.; Zhan, Q.; Mo, Y.; Sui, Q.; Li, Z. Optical Performance Monitoring of Multiple Parameters in Future Optical Networks. J. Lightwave Technol. 2021, 39, 3792-3800. [CrossRef]

8. Thyagaturu, A.S.; Mercian, A.; McGarry, M.P.; Reisslein, M.; Kellerer, W. Software defined optical networks (SDONs): A comprehensive survey. IEEE Commun. Surv. Tutor. 2016, 18, 2738-2786. [CrossRef]

9. Xie, J.; Yu, F.R.; Huang, T.; Xie, R.; Liu, J.; Wang, C.; Liu, Y. A survey of machine learning techniques applied to software defined networking (SDN): Research issues and challenges. IEEE Commun. Surv. Tutor. 2018, 21, 393-430. [CrossRef]

10. FG-ML5G, I. Focus Group on Machine Learning for Future Networks Including 5G. Available online: https://www.itu.int/en/ ITU-T/focusgroups/ml5g/Pages/default.aspx (accessed on 21 December 2021).

11. Pointurier, Y. Machine learning techniques for quality of transmission estimation in optical networks. J. Opt. Commun. Netw. 2021, 13, B60-B71. [CrossRef]

12. Saif, W.S.; Esmail, M.A.; Ragheb, A.M.; Alshawi, T.A.; Alshebeili, S.A. Machine learning techniques for optical performance monitoring and modulation format identification: A survey. IEEE Commun. Surv. Tutor. 2020, 22, 2839-2882. [CrossRef]

13. Tanaka, T.; Inui, T.; Kawai, S.; Kuwabara, S.; Nishizawa, H. Monitoring and diagnostic technologies usingdeep neural networks for predictive optical network maintenance. J. Opt. Commun. Netw. 2021, 13, E13-E22. [CrossRef]

14. Tanimura, T.; Hoshida, T.; Kato, T.; Watanabe, S.; Morikawa, H. Convolutional neural network-based optical performance monitoring for optical transport networks. J. Opt. Commun. Netw. 2019, 11, A52-A59. [CrossRef]

15. Wang, Z.; Yang, A.; Guo, P.; He, P. OSNR and nonlinear noise power estimation for optical fiber communication systems using LSTM based deep learning technique. Opt. Express 2018, 26, 21346-21357. [CrossRef] [PubMed]

16. Xiang, Q.; Yang, Y.; Zhang, Q.; Yao, Y. Joint, accurate and robust optical signal-to-noise ratio and modulation format monitoring scheme using a single Stokes-parameter-based artificial neural network. Opt. Express 2021, 29, 7276-7287. [CrossRef] [PubMed]

17. Zhao, Y.; Yu, Z.; Wan, Z.; Hu, S.; Shu, L.; Zhang, J.; Xu, K. Low Complexity OSNR Monitoring and Modulation Format Identification Based on Binarized Neural Networks. J. Lightwave Technol. 2020, 38, 1314-1322. [CrossRef]

18. Saif, W.S.; Alshawi, T.; Esmail, M.A.; Ragheb, A.; Alshebeili, S. Separability of histogram based features for optical performance monitoring: An investigation using t-SNE technique. IEEE Photonics J. 2019, 11, 1-12. [CrossRef]

19. Shen, T.S.R.; Meng, K.; Lau, A.P.T.; Dong, Z.Y. Optical performance monitoring using artificial neural network trained with asynchronous amplitude histograms. IEEE Photonics Technol. Lett. 2010, 22, 1665-1667. [CrossRef]

20. Cheng, Y.; Fu, S.; Tang, M.; Liu, D. Multi-task deep neural network (MT-DNN) enabled optical performance monitoring from directly detected PDM-QAM signals. Opt. Express 2019, 27, 19062-19074. [CrossRef]

21. Cheng, Y.; Zhang, W.; Fu, S.; Tang, M.; Liu, D. Transfer learning simplified multi-task deep neural network for PDM-64QAM optical performance monitoring. Opt. Express 2020, 28, 7607-7617. [CrossRef]

22. Jargon, J.A.; Wu, X.; Willner, A.E. Optical performance monitoring by use of artificial neural networks trained with parameters derived from delay-tap asynchronous sampling. In Proceedings of the 2009 Conference on Optical Fiber Communication, San Diego, CA, USA, 22-26 March 2009; pp. 1-3.

23. Khan, F.N.; Zhou, Y.; Sui, Q.; Lau, A.P.T. Non-data-aided joint bit-rate and modulation format identification for next-generation heterogeneous optical networks. Opt. Fiber Technol. 2014, 20, 68-74. [CrossRef]

24. Tan, M.C.; Khan, F.N.; Al-Arashi, W.H.; Zhou, Y.; Lau, A.P.T. Simultaneous optical performance monitoring and modulation format/bit-rate identification using principal component analysis. J. Opt. Commun. Netw. 2014, 6, 441-448. [CrossRef]

25. Fan, X.; Xie, Y.; Ren, F.; Zhang, Y.; Huang, X.; Chen, W.; Zhangsun, T.; Wang, J. Joint optical performance monitoring and modulation format/bit-rate identification by CNN-based multi-task learning. IEEE Photonics J. 2018, 10, 1-12. [CrossRef]

26. Khan, F.N.; Fan, Q.; Lu, C.; Lau, A.P.T. An optical communication's perspective on machine learning and its applications. J. Lightwave Technol. 2019, 37, 493-516. [CrossRef] 
27. Wang, D.; Wang, M.; Zhang, M.; Zhang, Z.; Yang, H.; Li, J.; Li, J.; Chen, X. Cost-effective and data size-adaptive OPM at intermediated node using convolutional neural network-based image processor. Opt. Express 2019, 27, 9403-9419. [CrossRef]

28. Luo, H.; Huang, Z.; Wu, X.; Yu, C. Cost-Effective Multi-Parameter Optical Performance Monitoring Using Multi-Task Deep Learning With Adaptive ADTP and AAH. J. Lightwave Technol. 2021, 39, 1733-1741. [CrossRef]

29. Saif, W.S.; Ragheb, A.M.; Alshawi, T.A.; Alshebeili, S.A. Optical Performance Monitoring in Mode Division Multiplexed Optical Networks. J. Lightwave Technol. 2020, 39, 491-504. [CrossRef]

30. Snyder, A.W.; Love, J. Optical Waveguide Theory; Springer Science \& Business Media: Berlin/Heidelberg, Germany, 2012.

31. Garth, S.J. Few-Mode Optical Waveguides and Their Study by the Four-Photon Mixing Process. Ph.D. Thesis, The Australian National University, Canberra, Australia, 1987.

32. Dods, S.D.; Anderson, T.B. Optical performance monitoring technique using delay tap asynchronous waveform sampling. In Proceedings of the Optical Fiber Communication Conference, Anaheim, CA, USA, 5-10 March 2006; p. OThP5.

33. Goodfellow, I.; Bengio, Y.; Courville, A. Deep Learning; MIT Press: Cambridge, MA, USA, 2016.

34. Olshausen, B.A.; Field, D.J. Sparse coding with an overcomplete basis set: A strategy employed by V1? Vis. Res. 1997, 37, 3311-3325. [CrossRef]

35. VPIphotonics. VPItransmissionMaker Optical Systems-Overview. Available online: https://www.vpiphotonics.com/Tools/ OpticalSystems / (accessed on 20 January 2022).

36. Vuong, J.; Ramantanis, P.; Frignac, Y.; Salsi, M.; Genevaux, P.; Bendimerad, D.F.; Charlet, G. Mode coupling at connectors in mode-division multiplexed transmission over few-mode fiber. Opt. Express 2015, 23, 1438-1455. [CrossRef] 